\title{
Exercise and Pharmacological Treatment: Influence on locomotor activity in SHR rats
}

\author{
NÁDIA E. SANTOS ${ }^{1} \mid$ CAMILA A. S. COELHO'| MILCA A. MORAIS'| BEATRIZ S. FRANCO'|ANDREA M. ESTEVES'
}

Faculdade de Ciências Aplicadas da Universidade Estadual de Campinas. Rua Pedro Zaccaria, 1300, Limeira - SP, Brazil, $13484-350$.

Correspondence to: Andrea M. Esteves,Faculdade de Ciências Aplicadas da Universidade Estadual de Campinas. Rua Pedro Zaccaria, 1300, Limeira - SP, Brazil, 13484-350.

email: andrea.esteves@fca.unicamp.br

https://doi.org/10.20338/bjmb.v13i1.123

\section{HIGHLIGHTS \\ - Treatment with PPX reduced locomotor activity on specific days. \\ -Exercise has been shown to cause changes in locomotor activity, similarly to PPX. \\ -Treatment with PPX for 48 days did not engender the augmentation effect in SHR rats.}

\section{ABBREVIATIONS}

OF open field

log transformation log

PE physical exercise

PLM Periodic Leg Movement

PPX pramipexole

PPX1 PPX $(0.1 \mathrm{mg} / \mathrm{kg})$ at 75 days of age

PPX2 PPX $(0.05 \mathrm{mg} / \mathrm{kg})$ at 91 days of age

PPX3 PPX (0.05 mg/kg) at 125 days of age

RLS Restless legs syndrome

SHR spontaneously hypertensive rats
BACKGROUND: Restless legs syndrome (RLS) is characterized by abnormal sensations in the lower limbs and negatively impacts on sleep. Dopaminergic agonists (pramipexole, PPX) may be used in the treatment. However, chronic use of these drugs may cause worsening of symptoms (augmentation). Physical exercise (PE) can also be effective in reducing RLS symptoms and improving sleep patterns.

AIM: The main purpose of this study was to evaluate the effect of PPX and acute PE on locomotor activity (RLS symptoms) of spontaneously hypertensive rats at different ages.

METHOD: Animals were distributed into 4 groups: Saline, PPX1 $(0.1 \mathrm{mg} / \mathrm{kg})$ started at 75 days of age, PPX2 $(0.05 \mathrm{mg} / \mathrm{kg})$ started at 91 days of age, and PPX3 $(0.05 \mathrm{mg} / \mathrm{kg})$ started at 125 days of age. The animals received a daily injection of PPX or saline (07:00 h) for 48 days. The PE (treadmill) consisted of a single session (30 $\mathrm{min}$ ) at 20-22 $\mathrm{m} / \mathrm{min}$, performed on day 49 . The evaluation of locomotor activity (open field) was performed at baseline and weekly, after administration of the drug.

RESULTS: Both PPX and PE caused changes in locomotor activity ( $\downarrow$ Total Ambulation, $\downarrow$ Rearing and $\uparrow$ Freezing), indicating reduction in RLS symptoms on specific days.

CONCLUSION: The groups receiving PPX did not present augmentation.

\section{INTRODUCTION}

Restless legs syndrome (RLS), classified as a sleep-related movement disorder, is a sensorimotor disorder, characterized by discomfort (lower limbs), with nocturnal worsening and relief with moving, generating a negative impact on sleep architecture and quality of life. ${ }^{1}$ Its prevalence, as well as the severity of symptoms may increase with age ${ }^{2}$, suggesting that the neurodegenerative process may play an important role in this syndrome. The life style of older adults as well as the senile changes, including cardiovascular and metabolism alterations, are also related to RLS. ${ }^{3}$ Moreover, patients with RLS may present Periodic Leg Movement (PLM), which is characterized by the rhythmic extension of the lower limbs, followed by ankle dorsiflexion, causing knee flexion and generalized motor activation in the lower limbs during sleep. PLM differs from RLS as it occurs involuntarily while the patient is sleeping. ${ }^{1}$

Treatment for RLS may be pharmacological, including dopaminergic agents, such 
as pramipexole $(\mathrm{PPX})^{4}$, or non-pharmacological, such as physical exercise $(\mathrm{PE})^{5}$. Moreover, scientific evidence demonstrates that the use of PPX may reduce locomotor activity in animal models, which is related to the reduction in RLS symptoms ${ }^{6,7} \mathrm{~A}$ study performed with C57BL / 6 mice with iron deprivation plus bilateral 6-hydroxydopamine lesions in the A11 nuclei (animal model of RLS) showed that acute and chronic treatment with PPX attenuated locomotor activity. ${ }^{7}$ Oliveira de Almeida et al. ${ }^{6}$ also observed that animals (Wistar) that received PPX presented a significant reduction in locomotor activity.

Although pharmacological treatment is effective in reducing symptoms, it may cause some adverse effects, such as augmentation, characterized by the anticipation of symptoms relative to the typical time of onset; or even, worsening of symptoms. Augmentation may occur when treatment is performed in the long-term, or with an increased dose. ${ }^{4}$ Augmentation has been reported in $60-85 \%$ of patients on levodopa treatment for 6 months or more ${ }^{8}$ and in $30 \%$ of patients taking a dopamine agonist, such as PPX, for at least 3 years. ${ }^{9}$ Studies suggest that this continues to occur for up to 9 years after treatment onset. ${ }^{10}$ On the other hand, in a more recent study ${ }^{11}$ conducted in Japanese patients with RLS, it was observed that short-term treatment with PPX $<46$ months) was a factor significantly associated with the presence of augmentation. It is not yet known if the incidence decreases after a longer duration of treatment, as well as which, it is not clear how rapidly augmentation develops or if it progresses over time to a more severe state. ${ }^{12}$

In previous studies that showed high rates of augmentation during PPX treatment, the mean daily dose ranged from 0.63 to $1.28 \mathrm{mg} /$ day..$^{9,13-15}$ Another study ${ }^{11}$ showed that the daily dose of PPX of $0.375 \mathrm{mg} /$ day was the optimal cutoff value to predict the occurrence of the augmentation symptom. Therefore, the daily dose of PPX should be kept low to prevent augmentation. However, it is not yet known whether decreasing the daily dose of PPX can prevent the presence of augmentation. ${ }^{11}$

In this sense, studies in animal models ${ }^{5,16}$, as well as in humans ${ }^{17}$, have shown the benefits of acute or chronic PE in reducing RLS symptoms. In addition, PE can improve sleep patterns, and represents a safe and low-cost treatment method.,16,17 Frank et al. ${ }^{5}$ observed that chronic treatment with PE (8 weeks) reduced the locomotor activity of spontaneously hypertensive rats (SHR), suggesting a reduction in RLS symptoms. Esteves et al. ${ }^{16}$ verified, in an animal model of RLS, a reduction in the number of limb movements after chronic exercise. In addition, Esteves et al. ${ }^{17}$ also demonstrated that PE may improve sleep patterns and reduce PLM levels in humans.

In the present study, the lineage of (SHR), composed of rats that developed attention deficit and hyperactivity disorder symptoms along with arterial hypertension, was used. This lineage has been shown to present characteristics related to sleep and behavior suggestive of sleep-related movement disorders, such as RLS. ${ }^{18}$

We believed that PPX treatment, as well as a PE session, could reduce locomotor activity and, consequently, RLS symptoms over time. In addition, it was expected that chronic use of PPX, as well as an increased dose, may cause augmentation of symptoms in SHR rats.

Thus, this study aimed to evaluate the effect of PPX treatment, as well as of one PE session, on the symptoms of RLS, by monitoring the locomotor activity, at different ages. In addition, we verified if there is worsening of symptoms (augmentation) with chronic treatment, or an increased dose of PPX. 


\section{METHODS}

The project was approved by the Universidade Estadual de Campinas (Unicamp) Ethics Committee (4559-1/17). The animals were distributed into 4 groups:

1) Saline $(n=4)$;

2) PPX (0.1 mg/kg) started at 75 days of age (PPX1) $(n=6)$;

3) PPX $(0.05 \mathrm{mg} / \mathrm{kg})$ started at 91 days of age (PPX2) $(n=4)$;

4) PPX (0.05 mg/kg) started at 125 days of age (PPX3) $(n=4)$.

As this study will present preliminary data (initial results), we used a small number of animals, and the sample size was not calculated.

\section{Experimental Design}

The animals were adapted to the open field (OF) test. After the adaptation period and baseline, treatment was started. Baseline assessments were performed according to the age at which the experiment was started in each group. Thus, for the PPX1 group, the baseline measurement was performed at 75 days, for the PPX2 group at 91 days, and for the PPX3 group at 125 days. Regarding the saline group, the baseline measurement was performed at 75 days. The animals were treated daily with PPX or saline, for 48 days, always at the same time (between 07:00 and 08:00 h). On day 37, the PPX dose was increased, and on day 49, one PE session was performed. The evaluation of locomotor activity was performed at baseline and weekly $(1,6,14,22,29,36,37,43$, and 49 days), shortly after (15 min) the PPX administration, or at the end of the PE session. Following the last OF test, the animals were euthanized (Figure 1).

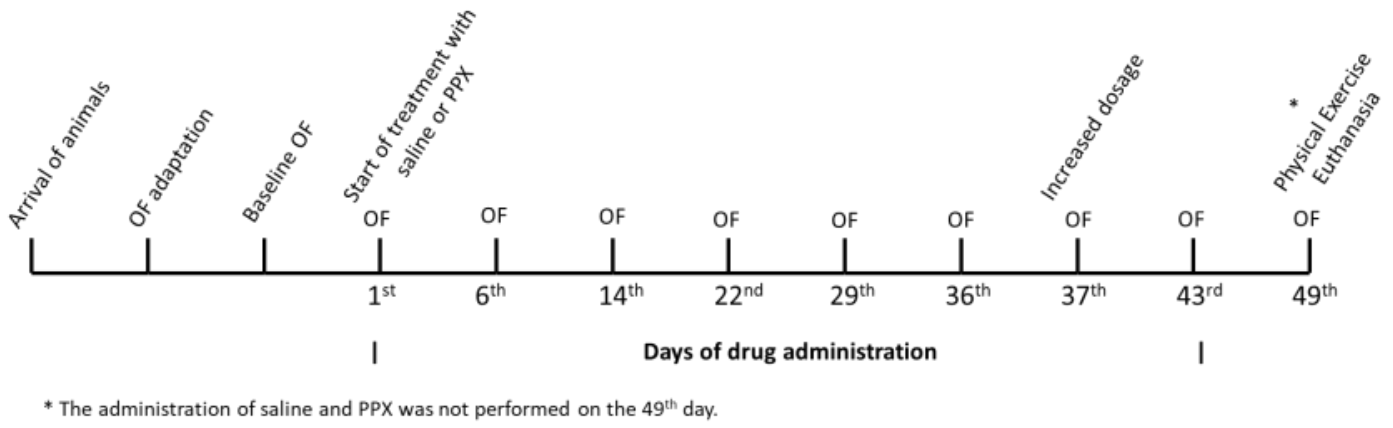

Figure 1. Experimental Design. OF: Open Field

\section{Experimental Procedure}

- Open field (OF)

Locomotor activity was evaluated in the OF, which consists of a circular arena of $81 \mathrm{~cm}$ (diameter), and 41-cm-high walls (open top). The lower part of the arena is divided into 19 quadrants, where the animals are placed individually for evaluation. Each animal remained in the test for $10 \mathrm{~min}$, and only the final five were evaluated. ${ }^{19}$ This type of assessment serves as an indirect parameter to measure the "desire to move," one of the most common symptoms in RLS. ${ }^{20}$

The analyzed parameters included total ambulation (the total number of quadrants that the animal stepped on with four paws); rearing frequency (the number of times that the animal supported itself with both hind legs); and freezing (total time the animal remained 
perfectly still, moving only the vibrissae). $\cdot{ }^{21}$ An increase in locomotor activity is related to an increase in RLS symptoms. Therefore, the higher the Total Ambulation and Rearing parameters, the greater the locomotor activity, and, consequently, the greater the symptoms of RLS. The reverse is also true. On the other hand, the lower the freezing time, the greater the locomotor activity, and, consequently, the greater the symptoms of RLS. The opposite is also true.

- Pharmacological Treatment

Pharmacological treatment with PPX lasted 48 days. For the PPX1 group, the dose was 0.1 (first 4 weeks) and $0.25 \mathrm{mg} / \mathrm{kg}$ (weeks 5 and 6). For the PPX2 and PPX3 groups, the dose was 0.05 (first 4 weeks) and $0.25 \mathrm{mg} / \mathrm{kg}$ (weeks 5 and 6). Each 0.1 $\mathrm{mg} / \mathrm{kg}$ was dissolved in $0.9 \%$ saline with the aid of a stirrer (Vortex-Genie $2 \AA$, Daigger $($, USA). Animals from the saline group received a saline vehicle at $0.9 \%(0.1 \mathrm{~mL} / 100 \mathrm{~g})$. The drug/saline was administered intraperitoneally between 07:00 and 08:00 h, daily. ${ }^{7}$ The animals were weighed weekly on a Precision Electronic Digital Scale for administration of the correct amount of the drug.

\section{Physical Exercise (PE)}

The PE session (treadmill) occurred on the final day of the experiment (day 49), at a speed of $21 \mathrm{~m} / \mathrm{min}$ (moderate intensity), which corresponds to approximately $60 \%$ of the maximal oxygen consumption. ${ }^{22}$ Exercise intensity was the same for all animals. The PE session started with a $5 \mathrm{~min}$ warm-up at $12-15 \mathrm{~m} / \mathrm{min}$, followed by $30 \mathrm{~min}$ at $20-22 \mathrm{~m} / \mathrm{min}$ (5), and took place between 07:00 and 08:00 $\mathrm{h}$.

\section{Statistical analysis}

Statistical analyses were performed using Statistica ${ }^{\circledR}$ for Windows $®$ (version 7.0, StatSoft Inc., Tulsa, OK). To analyze the behavioral parameters, the Kolmogorov Smirnov test was applied to verify the normality of the data. The distribution of the data was normalized by the transformation log $(\log )$. The open field and body mass results were analyzed by ANOVA for repeated measures (time - dependent variable \& groupindependent variable), followed by post hoc Tukey's test. The value of $a=5 \%$ was considered to be statistically significant. The data are expressed as mean \pm standard error.

\section{RESULTS}

Groups that received PPX presented alterations in locomotor activity after chronic use of the drug and the PE session. These changes were demonstrated through the total ambulance, rearing, and freezing parameters.

The PPX1 group (on days 1, 37, and 49), the PPX2 group (on day 37), and the PPX3 group (on day 37 ) presented significantly reduced total ambulation compared to the PPX1 group (at baseline and day 22), the PPX2 group (at baseline and days 22, 36, and 43), and the PPX3 group (at baseline and day 22, Figure 2A). All groups receiving PPX reduced rearing on days 1 and 37 compared to the baseline test (Figure 2B). Finally, the PPX1 group (on days 1, 37, and 49), the PPX2 group (on day 37) and the PPX3 group (on days 37 and 49 ) presented increased freezing compared to the baseline test and days 22 , 29,36 , and 43 (Figure 2C). 
The PPX1 and PPX3 groups presented alterations in body mass from week 2 of treatment in comparison to the baseline data of all groups (Figure 3). The PPX3 group demonstrated a significant increase in body mass (weeks 5 and 6 ) relative to the saline group at baseline, 1, 2, and 3 weeks. However, although not significant, the PPX3 group presented higher values of body mass than the saline group, at all studied moments after starting the drug treatment.
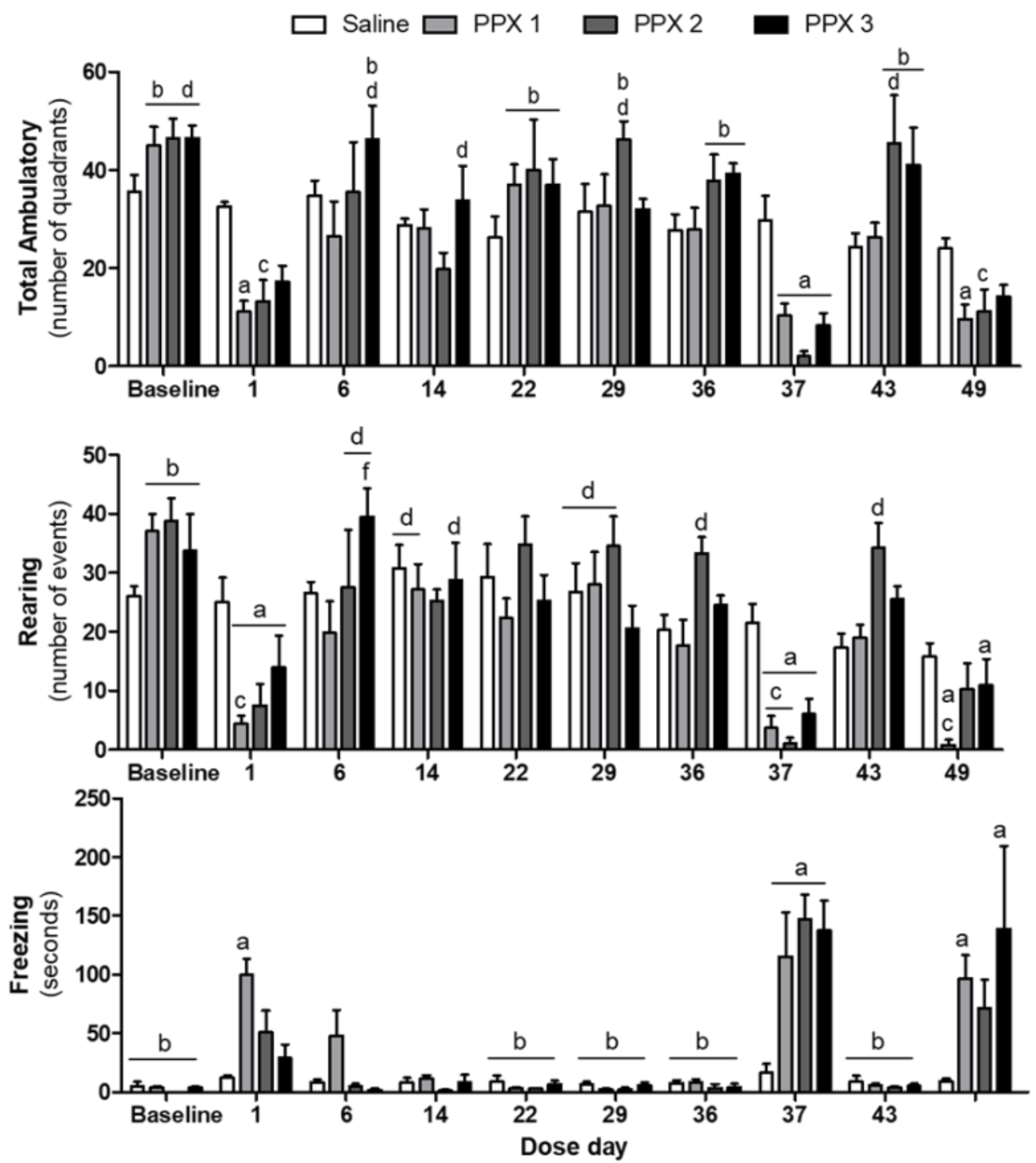

Figure 2. Parameters analyzed by the open field test of the animals in the saline group (control) and PPX groups at the ten assessment moments (baseline, 1, 6, 14, 22, 29, 36, 37, 43, and 49 days). ANOVA repeated measures, post hoc Tukey's test. (A) Total ambulatory: time $[F(9,126)=19.7772, p<0.00001]$; group $[F(3,126)$ $=2.6838, p<0.001]$; interaction $[F(27,126)=735.7397, p<0.00001]$; "a" differs from "b"; "c" differs from "d". (B) Rearing: time $[F(9,126)=26.4401, p<0.00001]$; group $[F(3,126)=3.4252, p<0.00001]$; interaction $[F(27,126)=335.9515, p<0.00001]$; "a" differs from "b"; "c" differs from "d". (C) Freezing: time $[F(9,126)=$ 21.9069, $p<0.00001]$; group $[F(3,126)=2.6523, p<0.00001]$; interaction $[F(27,126)=355.7966, p<$ 0.00001 ]; "a" differs from "b". 


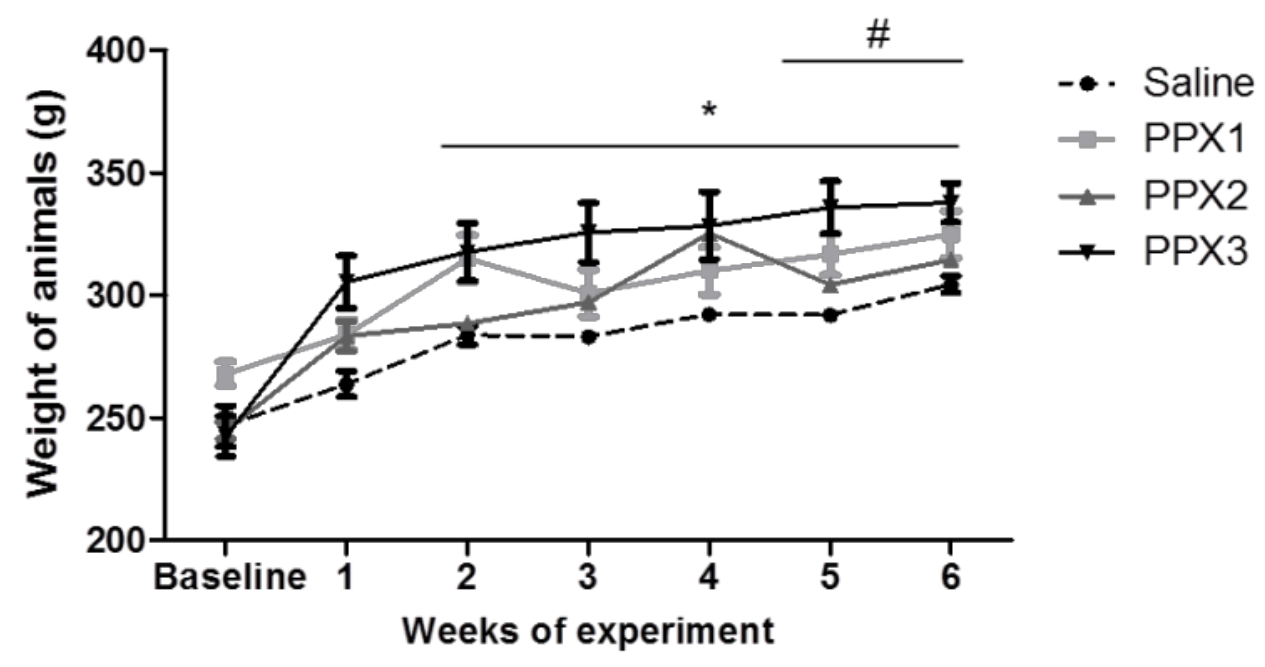

Figure 3. Body mass of the animals of the saline group (control) and PPX groups at baseline and during 6 weeks of treatment. ANOVA repeated measures, post hoc Tukey's test. Time $[F(6,84)=5.990, p<0.00001]$; group $[F(3,84)=3.456, p<0.0457]$; interaction $[F(18,84)=5.990 ; p<0.00001]$. ${ }^{*}$ PPX1 and PPX3 (on the 2nd, 3rd, 4th, 5th and 6th weeks) differs from baseline data of the saline, PPX1, PPX2, and PPX3 groups; \# PPX3 (5th and 6th weeks) differs from the saline group at baseline, 1, 2, and 3 weeks of experiment.

\section{DISCUSSION}

The present study aimed to evaluate the effect of chronic treatment with PPX, as well as of one PE session, on the symptoms of RLS, through evaluation of the locomotor activity, at different ages. Moreover, we aimed to check if there is worsening of symptoms (augmentation) with chronic treatment, or increased doses of PPX. The hypothesis of the study was that treatment with PPX, and a PE session, would reduce locomotor activity and, consequently, RLS symptoms, as age increased. In addition, it was expected that the chronic use of PPX, as well as increased doses, would cause the augmentation of symptoms in SHR rats.

Regarding the effect of PPX on locomotor activity, the total ambulation results were similar to the Rearing data, and all PPX groups presented lower values on days 1 (beginning of drug treatment) and 37 (dose increase) of treatment compared with the saline group. These findings reveal a reduction in locomotor activity and a possible decrease in RLS symptoms (days 1 and 37). Oliveira de Almeida et al. ${ }^{6}$ investigated the locomotor activity in Wistar rats after treatment with PPX (71 days), and observed a reduction in total ambulance (drug group) relative to both the basal period (days 1-57 of treatment) and the control group (days $8-43$ of treatment). Conversely, similar to observations in the present study, there was a reduction in rearing (drug group) compared with the control group, only on day 1 of treatment. Overall, these outcomes also suggest a decrease in RLS symptoms, but for a longer period than in the present study, possibly because the treatment with PPX was longer.

In addition, in our study, the freezing values increased in all PPX groups on days 1 and 37 of treatment, inferring less need for locomotion by the animals and, consequently, a reduction in the symptoms of RLS. Oliveira de Almeida et al. ${ }^{6}$ documented an increase in 
freezing (drug group) relative to both the basal period (days 1-64 of treatment) and the control group (days 1-43 and 57), thereby indicating reduced locomotor activity. However, it is worth mentioning that the PPX dose was constant and administered for a longer period (71 days) than in our study. In agreement with the present work, Oliveira de Almeida et al. ${ }^{6}$ did not observe augmentation of symptoms throughout the treatment.

A study ${ }^{7}$ that evaluated the effect of PPX treatment in another animal model of RLS noticed that the drug reduced locomotor activity and, similarly to our study, augmentation did not occur.

In the present study, different doses of PPX were used to verify if the effect of augmentation occurred. The dose choice of $0.1 \mathrm{mg} / \mathrm{kg}$ was based on the studies of Oliveira de Almeida et al. ${ }^{6}$ and Luo et al..$^{7}$ In both, changes in locomotor activity were observed in response to PPX treatment, but no manifestation of augmentation of symptoms. Thus, we decided to evaluate whether the treatment initiated with a lower dose of PPX $(0.05 \mathrm{mg} / \mathrm{kg})$ and with a subsequent increase to $0.25 \mathrm{mg} / \mathrm{kg}$ would cause alterations in locomotor activity, as well as augmentation of symptoms, since it is known that the effect of augmentation can be caused by the increased dose of dopaminergic agonists, over treatment.

In our study, the animals presented rapid habituation to the administration of pramipexole, where behavioral changes only occurred at the first dose and at the dose increase. This is the first study to evaluate whether augmentation occurs with the use of pramipexole in SHR rats. Therefore, more studies are needed to understand adaptation of the animals to different dosages and treatment periods.

Regarding the acute PE session, performed on the final day of the experiment (day 49), on which the animals did not receive PPX, results similar to the treatment with the drug were seen, with a reduction in total ambulation and rearing, and an increase in freezing. Thus, a single PE session promoted similar results to PPX treatment, reducing locomotor activity, indicating a decrease in RLS symptoms. Corroborating with our study, Esteves et al. ${ }^{17}$ noted a positive effect of acute aerobic PE in patients with PLM, concluding that PE may be a plausible alternative to pharmacological treatment, or as a complement to drug therapy in extreme cases.

Other research with animal models of RLS also demonstrated the beneficial effect of chronic PE on RLS/PLM symptoms ${ }^{5,16}$. Frank et al. ${ }^{5}$ analyzed the impact of chronic PE (treadmill) on locomotor activity (SHR rats). While those authors did not note any difference in total ambulation between the PE groups and the control, the PE groups presented lower rearing values.

One of the mechanisms that suggest improvement in sleep-related movement disorder symptoms through PE is related to the participation of the opioidergic and dopaminergic systems, which are highly stimulated during exercise. Esteves et al. ${ }^{17}$ observed a negative correlation between beta-endorphin levels released in plasma and PLM after an acute PE session ${ }^{17}$.

Regarding treatment with PPX, we illustrated an increase in the body mass of the animals of the PPX 1 and PPX 3 groups from week 2 when compared with the baseline data of all groups. Although not significant, the animals in the PPX3 group were heavier than the saline group, corroborating the findings of Oliveira de Almeida et al. ${ }^{6}$ in rats (Wistar) after chronic treatment with PPX. 


\section{CONCLUSION}

The groups that received PPX presented decreased locomotor activity compared with the saline group at the first dose (day 1), increased dose (day 37), and after the PE session (day 49). The PE session caused changes in locomotor activity similar to PPX, proving to be effective in reducing the symptoms of RLS. Furthermore, no augmentation of symptoms occurred with chronic treatment, or even with increasing doses of PPX. Further studies are needed to understand the augmentation of symptoms in SHR rats.

\section{REFERENCES}

1. Walters AS. Toward a better definition of the restless legs syndrome. The International Restless Legs Syndrome Study Group. Movement Disorders. 1995;10( 634-642).

2. Ohayon MM, Roth T. Prevalence of restless legs syndrome and periodic leg movement disorder in the general population. Journal of Psychosomatic Research. 2002;53(1):547554.

3. Cassel W, Kesper K, Bauer A, Grieger F, Schollmayer E, Joeres L. Significant association between systolic and diastolic blood pressure elevations and periodic limb movements in patients with idiopathic restless legs syndrome. Sleep medicine. 2016;17:109-120.

4. Hening WA, Allen RP, Earley CJ, Picchietti DL, Silber MH, Restless Legs Syndrome Task Force of the Standards of Practice Committee of the American Academy of Sleep M. An update on the dopaminergic treatment of restless legs syndrome and periodic limb movement disorder. Sleep. 2004;27(3):560-583.

5. Frank MK, de Mello MT, Lee KS, Daubian-Nose P, Tufik S, Esteves AM. Sleep-related movement disorder symptoms in SHR are attenuated by physical exercise and an angiotensin-converting enzyme inhibitor. Physiology \& behavior. 2016;154:161-168.

6. Oliveira de Almeida WA, Maculano Esteves A, Leite de Almeida-Junior C, et al. The effects of long-term dopaminergic treatment on locomotor behavior in rats. Sleep science. 2014;7(4):203-208.

7. Luo F, Li C, Ondo WG, Xu P, Xie W, Le W. The long-term effects of the dopamine agonist pramipexole in a proposed restless legs syndrome animal model. Sleep medicine. 2011;12(1):41-46.

8. Allen RP, Earley CJ. Augmentation of the restless legs syndrome with carbidopa/levodopa. Sleep. 1996;19(3):205-213.

9. Silber MH, Girish M, Izurieta R. Pramipexole in the management of restless legs syndrome: an extended study. Sleep. 2003;26(7):819-821.

10. Silver N, Allen RP, Earley CJ. Ten-year follow-up of efficacy and augmentation on pramipexole and methadone treatment of RLS. Sleep medicine. 2009;10(Suppl. 2):S27.

11. Takahashi M, Nishida S, Nakamur M, et al. Restless legs syndrome augmentation among Japanese patients receiving pramipexole therapy: Rate and risk factors in a retrospective study. PLoS One. 2017;12(3):e0173535. 
Brazilian Journal of Motor Behavior

12. Allen RP, Ondo WG, Ball E, et al. Restless legs syndrome (RLS) augmentation associated with dopamine agonist and levodopa usage in a community sample. Sleep medicine. 2011;12(5):431-439.

13. Jeon JY, Moon HJ, Song ML, Lee HB, Cho YW. Augmentation in restless legs syndrome patients in Korea. Sleep Breath. 2015;19(2):523 \pm 529

14. Silver N, Allen RP, Senerth J, Earley CJ. A 10-year, longitudinal assessment of dopamine agonists and methadone in the treatment of restless legs syndrome. Sleep medicine. 2011;12(5):440-444.

15. Lipford MC, Silber MH. Long-term use of pramipexole in the management of restless legs syndrome. Sleep medicine. 2012;13(10):1280 1285 .

16. Esteves AM, Lopes C, Frank MK, et al. Can physical exercise have a protective effect in an animal model of sleep-related movement disorder? Brain Research. 2016;1639:47-57.

17. Esteves AM, de Mello MT, Pradella-Hallinan M, Tufik S. Effect of acute and chronic physical exercise on patients with periodic leg movements. Medicine and science in sports and exercise. 2009;41(1):237-242.

18. Esteves AM, Lopes C, Frussa-Filho R, et al. Spontaneously hypertensive rats: possible animal model of sleep-related movement disorders. Journal of motor behavior. 2013;45(6):487-493.

19. Qu S, Le W, Zhang X, Xie W, Zhang A, W.G. O. Locomotion is increased in a11-lesioned mice with iron deprivation: a possible animal model for restless legs syndrome. Journal of Neuropathology \& Experimental Neurology 2007;66(5):383-388.

20. Allen RP, Chen C, Garcia-Borreguero D, et al. Comparison of pregabalin with pramipexole for restless legs syndrome. The New England journal of medicine. 2014;370(7):621-631.

21. Fukushiro DF, Calzavara MB, Trombin TF, et al. Effects of environmental enrichment and paradoxical sleep deprivation on open-field behavior of amphetamine-treated mice. Physiology \& behavior. 2007;92(4):773-779.

22. Arida RM, Scorza FA, dos Santos NF, Peres CA, Cavalheiro EA. Effect of physical exercise on seizure occurrence in a model of temporal lobe epilepsy in rats. Epilepsy research. 1999;37(1):45-52.

Citation: Santos NE, Coelho CAS, Morais MA, Franco BS, Esteves AM. Exercise and Pharmacological Treatment: Influence on locomotor activity in shr rats. BJMB.2019: 13(1): 23-31.

Editor: Dr Fabio Augusto Barbieri - São Paulo State University (UNESP), Bauru, SP, Brazil; Dr José Angelo Barela São Paulo State University (UNESP), Rio Claro, SP, Brazil; Dr Natalia Madalena Rinaldi - Federal University of Espírito Santo (UFES), Vitória, ES, Brazil.

Copyright: (C) 2019Santos, Coelho, Morais, Franco and Esteves and BJMB. This is an open-access article distributed under the terms of the Creative Commons Attribution- NonCommercial-NoDerivatives 4.0 International License which permits unrestricted use, distribution, and reproduction in any medium, provided the original author and source are credited.

Funding: This study was financed in part by the Coordenação de Aperfeiçoamento de Pessoal de Nível Superior Brasil (CAPES) - Finance Code 001.

Competing interests: The authors have declared that no competing interests exist.

DOl: $10.20338 /$ bjmb.v13i1.123 